\title{
Moving sidewinding forward: optimizing contact patterns for limbless robots via geometric mechanics
}

\author{
Baxi Chong*, Tianyu Wang*, Bo Lin*, Shengkai Li*, Grigoriy Blekherman*, Howie Choset ${ }^{\dagger}$ and Daniel I. Goldman* \\ ${ }^{*}$ Georgia Institute of Technology, ${ }^{\dagger}$ Carnegie Mellon University
}

\begin{abstract}
Contact planning is crucial to the locomotion performance of limbless robots. Typically, the pattern by which contact is made and broken between the mechanism and its environment determines the motion of the robot. The design of these patterns, often called contact patterns, is a difficult problem. In previous work, the prescription of contact patterns was derived from observations of biological systems or determined empirically from black-box optimization algorithms. However, such contact pattern prescription is only applicable to specific mechanisms, and is challenging to generalize. For example, the stable and effective contact pattern prescribed for a 12-link limbless robot can be neither stable nor effective for a 6-link limbless robot. In this paper, using a geometric motion planning scheme, we develop a framework to design, optimize, and analyze contact patterns to generate effective motion in the desired directions. Inspired by prior work in geometric mechanics, we separate the configuration space into a shape space (the internal joint angles), a contact state space, and a position space; then we optimize the function that couples the contact state space and the shape space. Our framework provides physical insights into the contact pattern design and reveals principles of empirically derived contact pattern prescriptions. Applying this framework, we can not only control the direction of motion of a 12-link limbless robot by modulating the contact patterns, but also design effective sidewinding gaits for robots with fewer motors (e.g., a 6-link robot). We test our designed gaits by robophysical experiments and obtain excellent agreement. We expect our scheme can be broadly applicable to robots which make/break contact.
\end{abstract}

\section{Introduction}

Limbless locomotion is achieved by properly coordinating body curvatures to interact with environment and generate selfpropulsion [28]. One of the most common limbless locomotion patterns is a traveling wave of body curvatures propagating from head to tail (lateral undulation) [17], which enables effective locomotion of most biological snakes. However, limited by the number of joints [19] and the availability of sensors [28], limbless robots often struggle when just using lateral undulation, especially in isotropic environments. Therefore, many robotic limbless locomotors use an alternative strategy of sidewinding, which generates robust and effective locomotion by regulating the body contact pattern (periodically making and breaking environmental contact) in addition to the lateral undulation [3, 4, 14, 22, 29]. Despite the advantages in making and breaking environmental contact, it comes with cost in additional degrees-of-freedom (DoF) to control the contact, which, if not properly controlled, can lead to unstable [7] or uncoordinated [22] locomotion.

Given the importance of contact pattern design, contact
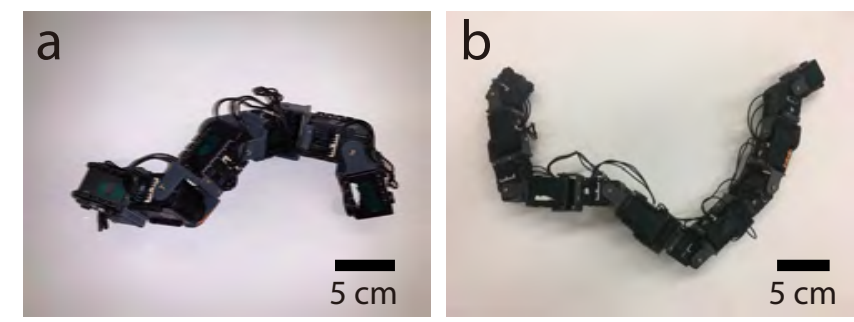

Fig. 1. Sidewinding limbless robot with varied number of motors. (a) A 6-link limbless robot, and (b) a 12-link limbless robot.

planning for limbless robots has been extensively researched [3, 7, 10, 22, 27]. A popular approach to contact pattern design is to take biological inspiration [9]. Specifically, contact planning is decomposed into two levels: in the higher level, the contact pattern is pre-determined using biologically inspired dynamics (e.g., the sinusoidal vertical wave [3]). At the lower level, the controller adapts the pre-determined contact patterns to the environment [4]. Another popular approach to contact pattern design is to use black-box controllers or algorithms [10] to optimize the contact patterns.

While existing approaches have demonstrated their efficacy in various robots, little is known about the principles behind contact planning. For example, it remains unclear why certain contact patterns are effective, and what happens if we perturb the contact pattern (e.g., delay the landing/lifting of some body segments). This lack of understanding of principles can limit our capability to (1) transfer contact planning insights gained in one robot to another, and (2) modulate the contact pattern to achieve high maneuverability (e.g., control the direction of motion, degree of rotation, etc) [3].

The field of geometric mechanics has been developed as a general scheme to link locomotor performance to arbitrary patterns of "self-deformation" [5, 15, 20, 25, 31, 33]. Specifically, the motion of a locomoting system is separated into a shape space (the internal joint angle space) and a position space (position and orientation of locomotor in the world frame). By establishing the mapping between velocities in shape and position spaces, geometric mechanics offers tools to allows us to visually analyze, design and optimize gaits. Despite recent efforts to extend geometric mechanics to mixed-contact systems [6, 8, 27], the scheme has limitations in designing and optimizing the contact patterns. 


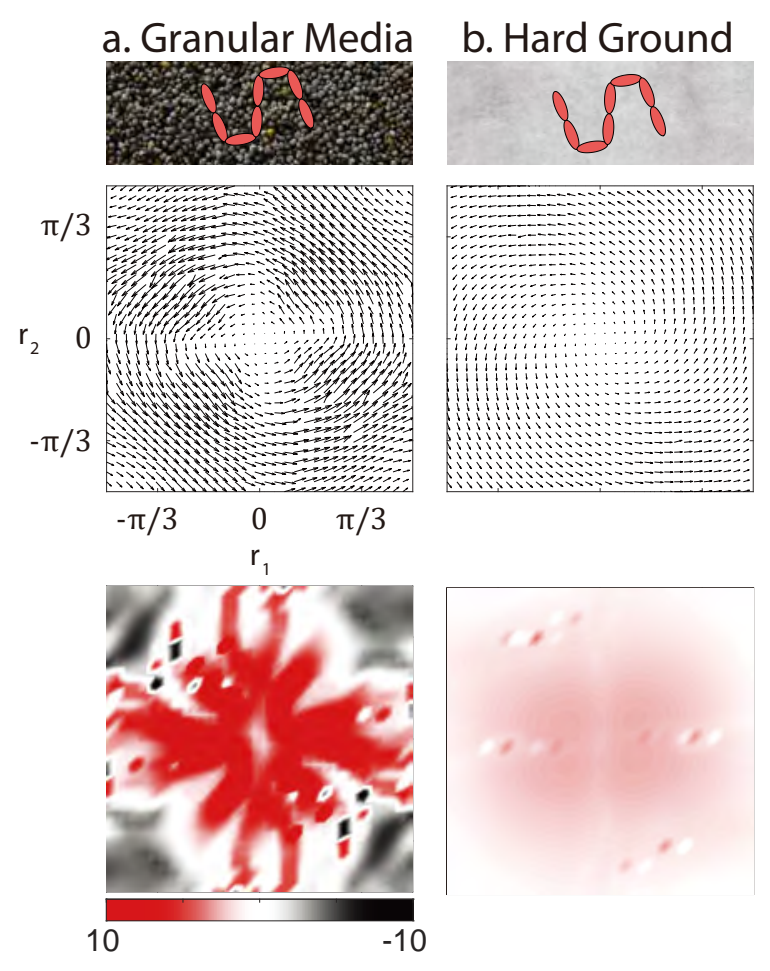

Fig. 2. Vector fields and height functions for an 8-link robot on granular media and hard ground with continuous contact. (a) The sketch, vector field, and height function for an 8-link robot moving on granular media (poppy seeds). The height function has a large magnitude. (b) The sketch, vector field, and height function for a 8-link robot moving on hard ground. The axes of all shape space are identical. The color bar of height functions in (a) and (b) are identical. The unit of color bar in height function is $B L / \pi^{2}$.

In this paper, we extend the geometric mechanics framework to design, analyze and optimize contact patterns in limbless sidewinding robots of arbitrary number of motors. One of our major contributions is to formulate contact pattern design problem using geometric mechanics framework. Specifically, we consider each contact state as an independent map connecting the shape space and position space. We then seek the optimal "switching" points between these maps that lead to the desired motion. Using the Hodge-Helmholtz theorem, we show that we can find the globally optimal transition points in the shape space. We then apply our framework to design gaits for limbless robots. As a result, we show that we can design effective sidewinding gait for robots with few motors (i.e., a 6-link limbless robot, Fig. 1 a) to achieve speed comparable to those with more motors (e.g., a 12-link limbless robot, Fig. 1 .b). In addition, we show that we can modulate the angle of motion (the angle of displacement w.r.t. the axis to of limbless robot) for a sidewinding limbless robot moving in isotropic environment. Our theoretical predictions are verified by robophysical experiments.

\section{Method}

\section{A. Geometric Mechanics}

In this subsection, we provide an overview of the geometric tools, which build the foundation of the framework introduced in this paper. For a more detailed and comprehensive review, we refer readers to [6, 12, 21, 35]. The geometric mechanics gait design framework separates the configuration space of a system into two spaces: a position space and a shape space. The position space represents the location (position and rotation) of a system in the world frame, while the shape space denotes the internal shape of the system. The geometric mechanics framework then establishes a functional relationship to map the velocities in the shape space to the velocities in the position space; this functional relationship is often called a local connection.

1) Kinematic Reconstruction Equation: In kinematic systems where inertial effects are negligible, the equations of motion $([21])$ can be approximated as:

$$
\xi=A(r) \dot{r}
$$

where $\boldsymbol{\xi}=\left[\xi_{x}, \xi_{y}, \xi_{\theta}\right]^{T}$ denotes the body velocity in the forward, lateral, and rotational directions; $\boldsymbol{r}$ denotes the internal shape variables (joint angles); $\boldsymbol{A}(\boldsymbol{r})$ is the local connection matrix, which encodes environmental constraints and the conservation of momentum. While there has been research extending geometric mechanics to higher dimensions [6, 26], the analysis and visualization power of geometric mechanics are particularly effective when the shape variable is 2-dimensional, i.e., $r \in \mathbb{R}^{2}$. In the applications where there are more than 2 joints (e.g. $N$ degrees-of-freedom), we use two shape basis functions [11] to reduce the dimensionality of the system:

$$
\boldsymbol{w}=\left[\boldsymbol{\beta}_{1}^{T}, \boldsymbol{\beta}_{2}^{T}\right] \boldsymbol{r},
$$

where $\boldsymbol{\beta}_{1}, \boldsymbol{\beta}_{2} \in \mathbb{R}^{N}$ are called the shape basis functions. In the application to snake robots, the shape basis functions are often chosen to be:

$$
\boldsymbol{\beta}_{1}(i)=\sin \left(2 \pi f_{s} \frac{i}{N-1}\right), \boldsymbol{\beta}_{2}(i)=\cos \left(2 \pi f_{s} \frac{i}{N-1}\right), 1 \leq i \leq N,
$$

where $f_{s}$ is the spatial frequency of the snake undulation, $i$ denotes the joint index, and $N$ is the total number of joints.

2) Numerical Derivation of Local Connection Matrix: The local connection matrix $\boldsymbol{A}$ can be numerically derived using resistive force theory (RFT) to model the ground reaction force [16, 32, 34]. In this section, we provide a brief derivation of the local connection matrix needed for this paper.

The ground reaction force (GRF) experienced by the locomotor is the sum of the GRF experienced by each body segment. RFT decomposes the resistive force experienced by a body segment of a locomotor into two components: thrust (perpendicular) and drag (parallel), i.e.,

$$
\boldsymbol{F}=\sum_{i \in I}\left(\boldsymbol{F}_{\|}^{i}+\boldsymbol{F}_{\perp}^{i}\right)
$$

where $\boldsymbol{F}_{\|}^{i}$ and $\boldsymbol{F}_{\perp}^{i}$ respectively denote forces parallel and perpendicular to the module $i ; I$ is the collection of all the modules that are instantaneously in contact with the environments. In many applications, the attack angle (the angle of body velocity w.r.t. the orientation of body segment) determines the $\boldsymbol{F}_{\|}$and $\boldsymbol{F}_{\perp}$ on this body segment, i.e., 


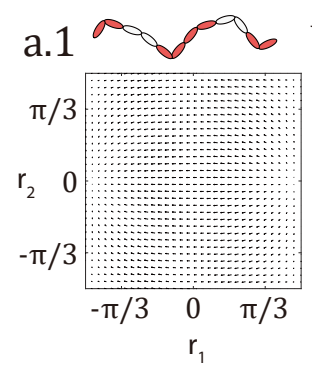

b.1
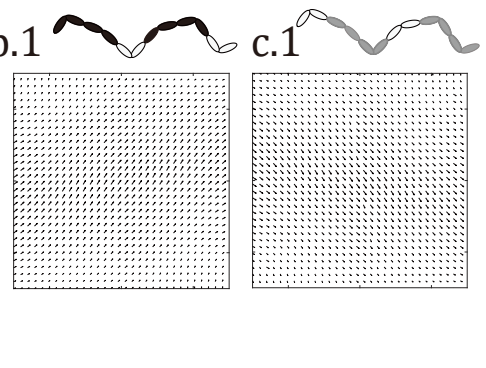

a.2

b.2
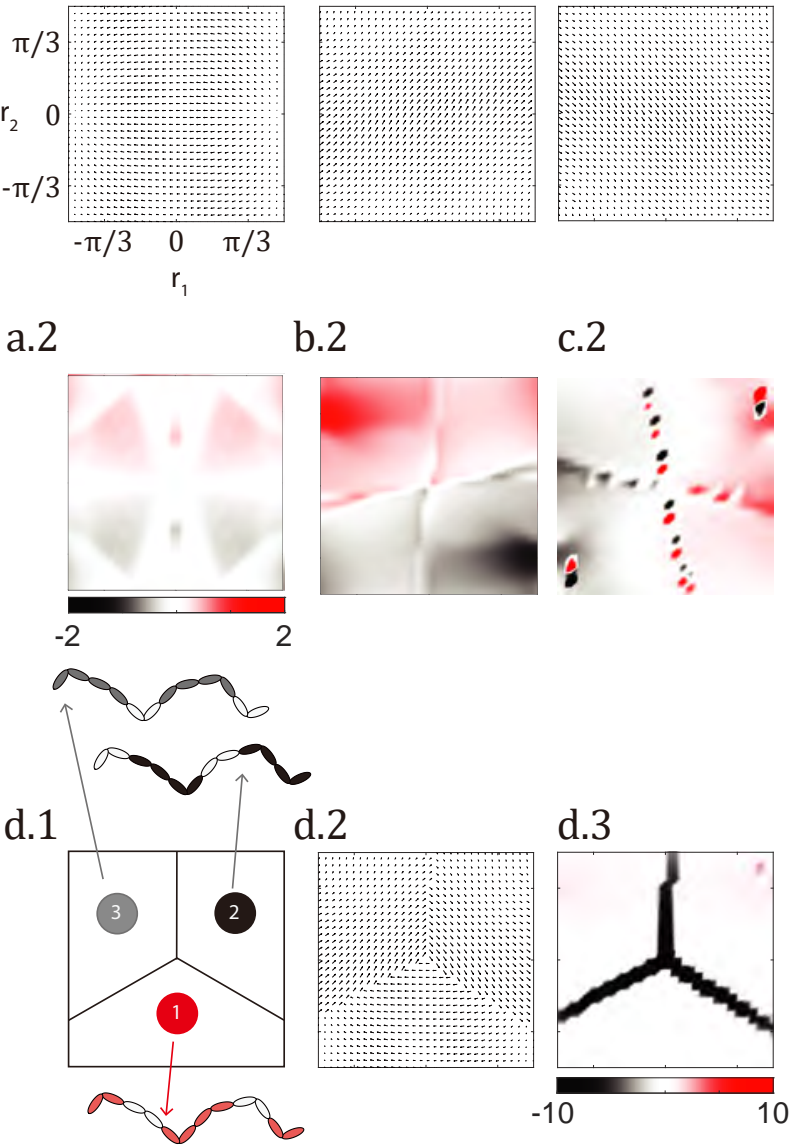

c. 2

b

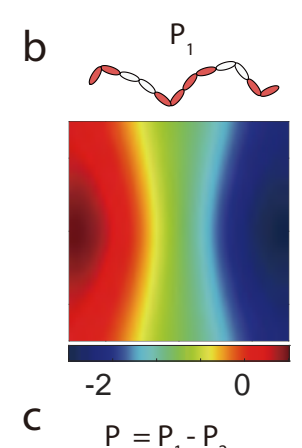

$-2$

C
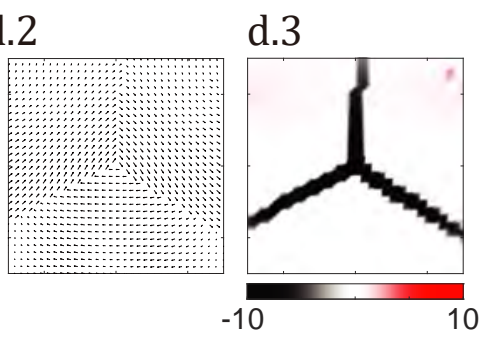

Fig. 3. Example of mixed contact pattern. (a-c) The vector fields and height functions for three contact states $I_{1}, I_{2}$, and $I_{3}$. Corresponding robot links which are in contact with the environment are denoted by red, black and grey. The color bar of height functions in (a), (b), and (c) are identical. (d.1) The contact pattern prescribed by Eq. 6. (d.2) The vector field prescribed by Eq. 77. (d.3) The corresponding height function. The axes of all shape space are identical. The unit of color bar in height function is $B L / \pi^{2}$.

$$
\boldsymbol{F}_{\|}=\boldsymbol{F}_{\|}(\chi), \boldsymbol{F}_{\perp}=\boldsymbol{F}_{\perp}(\chi),
$$

where $\chi$ is the attack angle. $\boldsymbol{F}_{\|}$and $\boldsymbol{F}_{\perp}$ are approximately independent of the magnitude of the velocity in granular and frictional systems [34]. Depending on the substrate, we can choose the corresponding RFT functions to approximate the ground reaction forces.

The attack angles $\chi$ of each segment can be calculated from the body velocity $\xi$, body shape $\boldsymbol{r}$, and shape velocity $\boldsymbol{r}$ [24]. By assuming quasi-static motion, we consider the total net force applied to the system is zero at any instant in time:

$$
\boldsymbol{F}=\sum_{i \in I}\left[\boldsymbol{F}_{\|}^{i}(\boldsymbol{\xi}, \boldsymbol{r}, \dot{\boldsymbol{r}})+\boldsymbol{F}_{\perp}^{i}(\boldsymbol{\xi}, \boldsymbol{r}, \dot{\boldsymbol{r}})\right]=0
$$

At a given body shape $\boldsymbol{r}$, Eq.(2) connects the shape velocity $\dot{r}$ to the body velocity $\xi$. Therefore, by implicit function theorem and the linearization process, we can numerically derive
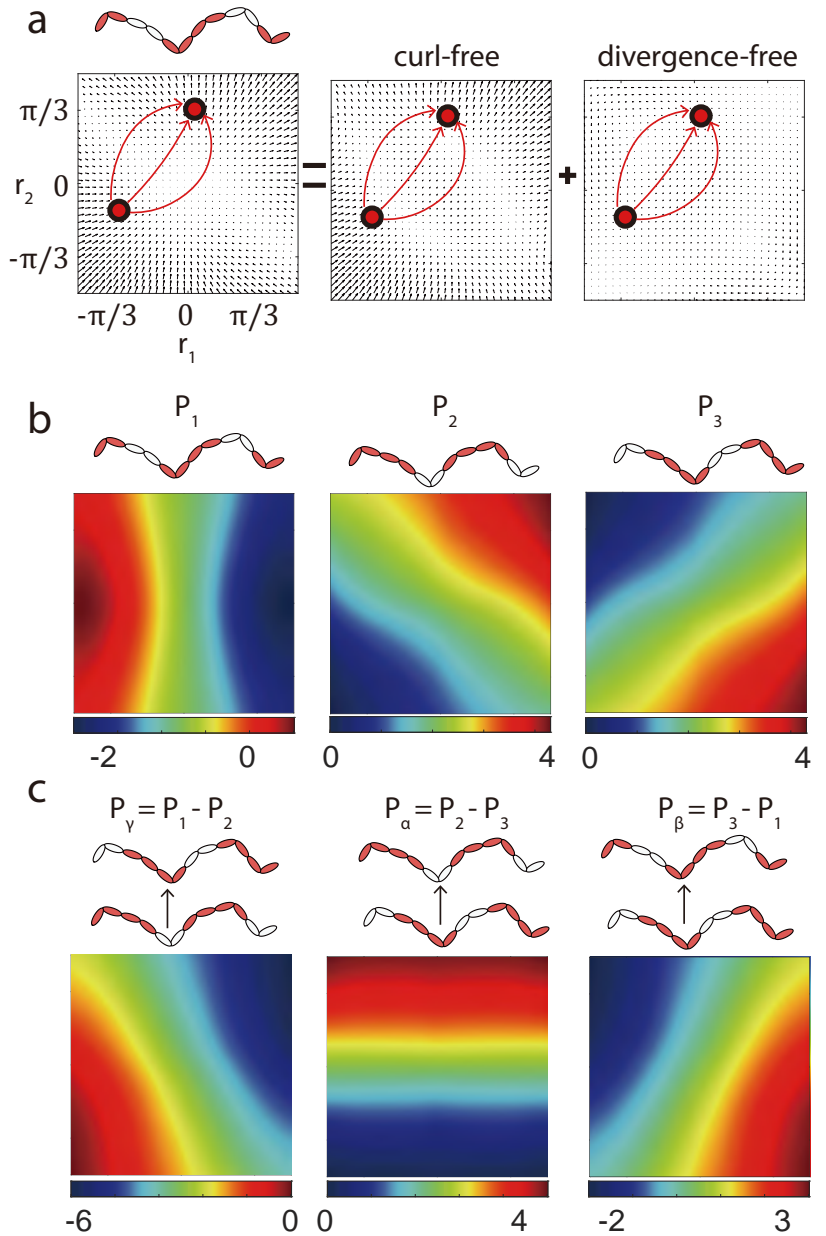

Fig. 4. Illustration of a contact pattern optimization. (a) The vector field and its curl-free component and divergence-free component by the HodgeHelmholtz decomposition. (b) The potential functions for $P_{1}, P_{2}$ and $P_{3}$. Note that, in curl-free components, the line integral is path-independent, allowing us to compute the potential function to estimate the line integral between any points. (c) The potential function difference for $P_{\gamma}=P_{2}-P_{1}, P_{\alpha}=P_{3}-P_{2}$, and $P_{\beta}=P_{1}-P_{3}$. The axes of all shape spaces are identical.

the local connection matrix $\boldsymbol{A}(\boldsymbol{r})$. In our implementation, we compute the solution of Eq.(2) using the MATLAB function fsolve.

3) Connection Vector Fields and Height Functions: Each row of the local connection matrix $\boldsymbol{A}$ corresponds to a component direction of the body velocity. Each row of the local connection matrix, over the shape space, then forms a connection vector field. In this way, the body velocities in the forward, lateral, and rotational directions are computed as the dot product of connection vector fields and the shape velocity $\dot{r}$.

The displacement along the gait path $\partial \phi$ can be obtained by integrating the ordinary differential equation ([13]) below:

$$
g(T)=\int_{\partial \phi} T_{e} L_{g(\boldsymbol{r})} A(\boldsymbol{r}) \mathrm{d} \boldsymbol{r}
$$

where $g(\boldsymbol{r})=[x(\boldsymbol{r}), y(\boldsymbol{r}), \alpha(\boldsymbol{r})]^{T}$ represents the position and rotation of body frame viewed in the world frame at position 
$r$ [24], $T$ is the time period of a gait cycle, and $g(T)=$ $[\Delta x, \Delta y, \Delta \alpha]^{T}$ denotes the translation and rotation of the body frame (w.r.t. the world frame) in one gait cycle. Note that $T_{e} L_{g}$ is the left-lifted action with respect to the coordinates of $g$ :

$$
T_{e} L_{g}=\left[\begin{array}{ccc}
\cos (\alpha) & -\sin (\alpha) & 0 \\
\sin (\alpha) & \cos (\alpha) & 0 \\
0 & 0 & 1
\end{array}\right] .
$$

The integral of Eq. (3) can be approximated to the first order by:

$$
\left(\begin{array}{c}
\Delta x \\
\Delta y \\
\Delta \theta
\end{array}\right)=\int_{\partial \phi} \boldsymbol{A}(\boldsymbol{r}) \mathrm{d} \boldsymbol{r}=\int_{\partial \phi}\left[\begin{array}{c}
\boldsymbol{A}^{x}(\boldsymbol{r}) \\
\boldsymbol{A}^{y}(\boldsymbol{r}) \\
\boldsymbol{A}^{\theta}(\boldsymbol{r})
\end{array}\right] \mathrm{d} \boldsymbol{r},
$$

where $\boldsymbol{A}^{x}, \boldsymbol{A}^{y}, \boldsymbol{A}^{\theta}$ are the three rows of the local connections respectively. The accuracy of the approximation in Eq. (4) can be optimized by properly choosing the body frame [13, 18]. According to Stokes' Theorem, the line integral along a closed curve $\partial \phi$ is equal to the surface integral of the curl of $\boldsymbol{A}(\boldsymbol{r})$ over the surface enclosed by $\partial \phi$ :

$$
\int_{\partial \phi} \boldsymbol{A}(\boldsymbol{r}) \mathrm{d} r=\iint_{\phi} \nabla \times \boldsymbol{A}(\boldsymbol{r}) \mathrm{d} r_{1} \mathrm{~d} r_{2},
$$

where $\phi$ denotes the surface enclosed by $\partial \phi$. The curl of the connection vector field, $\nabla \times \boldsymbol{A}(\boldsymbol{r})$, is referred to as the height function [13]. The three rows of the vector field $\boldsymbol{A}(\boldsymbol{r})$ can thus produce three height functions in the forward, lateral, and rotational direction respectively.

With the above derivation, the gait design problem is simplified to drawing a closed path in the shape space. Displacement can be approximated by the integral of the surface enclosed by the gait path. Hence, the maximization of the integral leads to the maximization of displacement.

4) Effect of Drag Anisotropy: Locomotion effectiveness can be highly dependent on the ground reaction force. Specifically, while limbless robots can achieve good mobility on granular media using lateral undulation, they often struggle on hard ground [2]. We compare the height function for an 8-link snake robot (with $f_{s}=1.5$ ) moving on surface of a model granular media (poppy seeds) and hard ground (Fig. 2).

The ground reaction forces governing the interaction of body segments and granular media are well studied when moving on a granular surface. The forces $\boldsymbol{F}_{\perp}$ and $\boldsymbol{F}_{\|}[23$ can be approximated by:

$$
f_{\perp}=C \sin (\chi), f_{\|}=A \cos (\chi)+B(1-\sin (\chi))+F_{0},
$$

where $\chi$ is the attack angle; $C=0.66, A=0.27, B=-0.32$, $F_{0}=0.09$ is the empirically fitted function to characterize the granular media resistance force [1, [30]. From structure and magnitude of its height function (Fig. 2 a), we see that, with proper gaits, the robot can move effectively on granular media as discussed in [30].

The ground reaction force between the body segments and the hard ground can then be modelled by dry Coulomb kinetic friction: $\quad f_{\perp}=f_{0} \sin (\chi), f_{\|}=f_{0} \cos (\chi)$, where $f_{0}=\mu F$ is the magnitude of the Coulomb kinetic friction, $\mu$ is the coefficient of friction and $F$ is the magnitude of the normal supporting force. The height function (Fig. 2 b) suggests that the robot has almost negligible speed regardless of the choices of gaits. However, it is important for limbless robots to move effectively on hard ground. Inspired by the sidewinding snakes [28], limbless robots can greatly improve the maneuverability by properly controlling their contact patterns [3].

\section{B. Contact Scheduling}

In the previous section, because dealing with lateral undulation and continuous contact, we made an assumption that there is no change in contact states throughout the gait. In other words, $I$ is independent of $\boldsymbol{r}$. In this section, we show that if we relax this assumption and incorporate a second wave, we can greatly improve the maneuverability of locomotion.

1) Single Contact State: Consider a 12-link limbless robot moving on hard ground. We assign a binary variable to each link, $c(i)$, such that $c(i)=0$ denotes link $i$ in swing phase (no contact) and $c(i)=1$ denotes link $i$ in stance phase (full contact). Therefore, $I=\{i \mid c(i)=1\}$. As we will discuss in Sec. III.A, the structure of the robot restricted that $c(2 i)=$ $c(2 i-1)$, i.e., the contact state between two consecutive vertical joints are identical.

We consider three distinct contact states: $I_{1}, I_{2}$, and $I_{3}$ :

\begin{tabular}{|c|c|c|c|c|c|c|}
\hline Contact state & $c(1)$ & $c(3)$ & $c(5)$ & $c(7)$ & $c(9)$ & $c(11)$ \\
& $c(2)$ & $c(4)$ & $c(6)$ & $c(8)$ & $c(10)$ & $c(12)$ \\
\hline$I_{1}$ & 1 & 0 & 1 & 1 & 0 & 1 \\
\hline$I_{2}$ & 1 & 1 & 0 & 1 & 1 & 0 \\
\hline$I_{3}$ & 0 & 1 & 1 & 0 & 1 & 1 \\
\hline
\end{tabular}

The choice of the contact states $I_{i}$ is based on the stability and the symmetry of the systems, such that two thirds of the modules are on the ground. Note that none of these contact states are dependent on $\boldsymbol{r}$. Their realizations can be visualized in Fig. 3. (a-c). For each contact state, we compute its vector field and height function in lateral direction (Fig. 3 (a-c)). We observe that in all the cases, the height functions do not have regular patterns and their magnitude is low, which indicates limited mobility when a limbless robot uses a single contact state.

2) Mixed Contact State: Although each individual contact state cannot lead to effective displacement, previous work demonstrated that their combination can enable new motion behaviors. In this section, we evaluate the locomotion performance of mixed contact state.

Inspired by [27], we construct the contact state as:

$$
I\left(r_{1}, r_{2}\right)=\left\{\begin{array}{ll}
I_{1}, & \text { if } \operatorname{atan} 2\left(r_{2}, r_{1}\right) \in(7 \pi / 6,11 \pi / 6] \\
I_{2}, & \text { if } \operatorname{atan} 2\left(r_{2}, r_{1}\right) \in(\pi / 2,7 \pi / 6] \\
I_{3}, & \text { if } \operatorname{atan} 2\left(r_{2}, r_{1}\right) \in(-\pi / 6, \pi / 2]
\end{array},\right.
$$

where $\operatorname{atan} 2$ is the four-quadrant inverse tangent operator. In this way, we can rewrite the local connection as: 


$$
\boldsymbol{A}\left(r_{1}, r_{2}\right)=\left\{\begin{array}{ll}
\boldsymbol{A}_{1}, & \text { if } \operatorname{atan} 2\left(r_{2}, r_{1}\right) \in(7 \pi / 6,11 \pi / 6] \\
\boldsymbol{A}_{2}, & \text { if } \operatorname{atan} 2\left(r_{2}, r_{1}\right) \in(\pi / 2,7 \pi / 6] \\
\boldsymbol{A}_{3}, & \text { if } \operatorname{atan} 2\left(r_{2}, r_{1}\right) \in(-\pi / 6, \pi / 2]
\end{array} .\right.
$$

Its realization is shown in Fig. 3d.1. We then obtain the vector field and height function using Eq. (6) in Fig. 3 d(2-3). Interestingly, the new height function has high magnitude and exhibits regular patterns (dark region along the boundary).

\section{Optimal Contact Scheduling}

Note that Eq. (6) is manually designed, inspired by biology and empirical experience [27]. Thus, the optimality of Eq. (6) remains unclear. To explore the optimization of contact patterns, we formulate the following optimization problem. To simplify our problem, we limit the number of contact states to be 3 .

Problem 1. Given 3 vector fields $\boldsymbol{A}_{1}^{x}, \boldsymbol{A}_{2}^{x}, \boldsymbol{A}_{3}^{x}$ in a shape space $M$, let $p$ be any partition $M=M_{1}^{p} \cup M_{2}^{p} \cup M_{3}^{p}$ and it induces the vector field $\boldsymbol{A}_{p}^{x}(\boldsymbol{r})$ such that for $i=1,2,3$,

$$
\boldsymbol{A}_{p}^{x}(\boldsymbol{r})=A_{i}^{x}(\boldsymbol{r}) \text { if } \boldsymbol{r} \in M_{i}^{p} .
$$

Let $L^{p}$ be the set of closed loops $l$ in $M$ such that for $i=1,2,3$, and assume that the intersection $l \cap M_{i}^{p}$ is simply connected. The optimization problem is

$$
\max _{l, p} \oint_{l} \boldsymbol{A}_{p}^{x}(\boldsymbol{r}) \mathrm{d} \boldsymbol{r} \forall l \in L^{p} .
$$

Since each $l \cap M_{i}^{p}$ is simply connected, any two of them have a unique intersection point. We may define the following:

$$
\begin{aligned}
l_{1} & =l \cap M_{1}^{p}, & l_{2} & =l \cap M_{2}^{p}, \\
\left\{q_{\beta}\right\} & =l_{1} \cap l_{3}, & l_{3} & =l \cap M_{3}^{p} \\
& =l_{1} \cap l_{2}, & \left\{q_{\alpha}\right\} & =l_{2} \cap l_{3} .
\end{aligned}
$$

Then

$$
\oint_{l} \boldsymbol{A}(\boldsymbol{r}) \mathrm{d} \boldsymbol{r}=\sum_{i=1}^{3} \int_{l_{i}} \boldsymbol{A}_{i}^{x}\left(r_{1}, r_{2}\right) \mathrm{d} \boldsymbol{r} .
$$

Note that in Eq. 9), each component is path-dependent, which is not desirable. From the Hodge-Helmholtz theorem, any vector field can be decomposed into the sum of a curl-free component, $\left(\boldsymbol{A}_{1}^{x}\right)_{c}$, and a divergence-free component, $\left(\boldsymbol{A}_{1}^{x}\right)_{d}$. In other words,

$$
\boldsymbol{A}_{1}^{x}=\left(\boldsymbol{A}_{1}^{x}\right)_{c}+\left(\boldsymbol{A}_{1}^{x}\right)_{d}
$$

Note that in our applications, the curl-free component has a much greater magnitude than the divergence-free component (Fig. 4 a). Therefore, we approximate the line integral in the original vector field by the line integral in the curlfree component from Hodge-Helmholtz decomposition. Note that in the case where the divergence-free component has comparable magnitude as the curl-free component, we can use the divergence-free components to determine the paths connecting the intersections once we determined the partition.
For curl-free vector fields the line-integral is pathindependent. Suppose the corresponding potential functions of the curl-free components are $P_{1}^{x}, P_{2}^{x}, P_{3}^{x}$, respectively (Fig. 4b) By the Fundamental Theorem of Calculus, we have

$$
\int_{l_{1}} \boldsymbol{A}_{1}^{x}\left(r_{1}, r_{2}\right) \mathrm{d} \boldsymbol{r} \approx \int_{l_{1}}\left(\boldsymbol{A}_{1}^{x}\right)_{c}\left(r_{1}, r_{2}\right) \mathrm{d} \boldsymbol{r}=P_{1}^{x}\left(q_{\beta}\right)-P_{1}^{x}\left(q_{\gamma}\right) .
$$

The other two terms in 9 are decomposed similarly. Then our objective function becomes

$$
\begin{aligned}
\oint_{l} \boldsymbol{A}(\boldsymbol{r}) \mathrm{d} \boldsymbol{r} & \approx\left(P_{1}^{x}\left(q_{\beta}\right)-P_{1}^{x}\left(q_{\gamma}\right)\right)+\left(P_{2}^{x}\left(q_{\gamma}\right)-P_{2}^{x}\left(q_{\alpha}\right)\right) \\
& +\left(P_{3}^{x}\left(q_{\alpha}\right)-P_{3}^{x}\left(q_{\beta}\right)\right) \\
& =\left(P_{3}^{x}-P_{2}^{x}\right)\left(q_{\alpha}\right)+\left(P_{1}^{x}-P_{3}^{x}\right)\left(q_{\beta}\right)+\left(P_{2}^{x}-P_{1}^{x}\right)\left(q_{\gamma}\right) \\
& =P_{\alpha}^{x}\left(q_{\alpha}\right)+P_{\beta}^{x}\left(q_{\beta}\right)+P_{\gamma}^{x}\left(q_{\gamma}\right),
\end{aligned}
$$

where $P_{\alpha}^{x}:=P_{3}^{x}-P_{2}^{x}, P_{\beta}^{x}:=P_{1}^{x}-P_{3}^{x}$, and $P_{\gamma}^{x}:=P_{2}^{x}-P_{1}^{x}$ are the potential function difference (PFD) (Fig. 4 $\mathrm{c}$ c). Note that our objective function has separated parameters - the coordinates of $q_{\alpha}, q_{\beta}, q_{\gamma}$. In addition, the choices of $p$ and $l$ imply that all 3 intersection points could be arbitrary points in $M$. As a result, when (10) is optimized, so are the 3 individual terms in (10). Therefore, $q_{\alpha}$ is the point in $M$ that optimizes the univariate function $P_{\alpha}^{x}$. Parameters $q_{\beta}, q_{\gamma}$ are characterized similarly.

Since the vector fields $\boldsymbol{A}^{x}(r)$ are given, so are the PFDs $P_{\alpha}^{x}, P_{\beta}^{x}, P_{\gamma}^{x}$. Thus we can find the optimal contact scheduling by solving these 3 individual optimization problems. In practice, if we discretize values of $P_{\alpha}^{x}, P_{\beta}^{x}, P_{\gamma}^{x}$, we can apply numerical algorithms to solve these optimization problems.

Once $q_{\alpha}, q_{\beta}, q_{\gamma}$ are found, we can then choose a generic point $q_{0}$ (in practice, $q_{0}$ can be chosen to be the origin) in $M$, and extend a curve connecting $q$ and $q_{\alpha}$ to the boundary of $M$, which serves as the boundary between $M_{2}^{p}$ and $M_{3}^{p}$. The other two boundaries are obtained by connecting and extending $q, q_{\beta}$ and $q, q_{\gamma}$, and we obtain the partition $p$, which leads to the optimal contact scheduling.

Note that our methods can be applied to systems with more than 3 states. Consider an undirected graph $G(V, E)$, where $v_{i} \in V$ denotes a contact state $i$, and $\left\{v_{i}, v_{j}\right\} \in E$ denotes that the transition from contact state $i$ to contact state $j$ is feasible. Then for each cycle, $o_{i}$, in $G$, we can use our method to determine its optimal transition points in the shape space $Q\left(o_{i}\right)$ and the corresponding displacement $D\left(o_{i}\right)$. Then the optimal in $G$ is then $\max _{i} D\left(o_{i}\right)$.

\section{Results}

\section{A. Experimental Setup}

We carried out robophysical experiments with a modular limbless robot composed of identical actuated modules (Dynamixel AX-12A servo motors). The number of modules varied according to the gait tested, but the arrangement of modules always followed the convention that the rotation axes of two neighboring modules were perpendicular to each other (Fig. 11. Thus, all rotation axes of odd modules lie in the same 
plane and the same is true for even modules. In experiments, odd modules were actuated to control the body shape in the horizontal plane and even modules were for the vertical plane.

Experiments were conducted on flat, hard ground, with the assumption that the ground reaction forces are given by dry Coulomb kinetic friction with friction coefficient $\mu=$ $0.35 \pm 0.06$. The joints of the robot were controlled by direct joint angle set-point commands. We conducted three repeat experiments for each gait we tested. In a single experiment, the robot executed three complete gait cycles.

To track the robot's motion in its position space, 6 IR reflective markers were attached along the body of the robot with equal distance along the body. An OptiTrack motion capture system with 6 OptiTrack Flex 13 cameras was used. 3D positions of the markers were tracked at 120 FPS frame rate. The motions of the robot executing 3 cycles of gait were collected from the robot formed the first configuration until the robot reached the last configuration.

We measured the angle of motion and displacement from the robot motion data we collected. To calculate the angle of motion for one experiment ( 3 complete gait cycles), we selected the first and the last sets of marker positions, which correspond to the initial and the ending robot configurations, respectively. We then calculated the averaged geometric center of the body for two configurations by taking the mean of the position of markers. Thus, the trajectory of the geometry center in the world frame can be determined by connecting the starting and ending geometric centers. Similarly, the angle of motion can be measured by the angular difference between the geometry center trajectory and the horizontal axes (perpendicular to the starting body axis). The displacement can also be measured by the projection of the geometry center trajectory onto the horizontal axes.

\section{B. Modulating Angle of Motion}

In previous work [3, 7], the sidewinding gait for limbless robots is decomposed into two waves in the horizontal plane and the vertical plane, respectively. In this way, the formulas of locomotion are prescribed as:

$$
\begin{gathered}
\theta(2 j-1, t)=A_{h} \sin \left(2 \pi K \frac{2 j-1}{N}+2 \pi f t\right), \\
\theta(2 j, t)=A_{v} \sin \left(2 \pi K \frac{2 j}{N}+2 \pi f t+\phi_{0}\right),
\end{gathered}
$$

where $j=1,2, \ldots, N / 2 ; \theta(2 j-1, t)$ and $\theta(2 j, t)$ refer to the yaw joint angles and the pitch joint angles respectively; $K$ is the spatial frequency of the sidewinding gaits; $A_{h}$ and $A_{v}$ are the amplitude of the horizontal wave and the vertical wave respectively; $f$ defines the temporal frequency; and $\phi_{0}$ is the phase lag between the horizontal and the vertical waves.

Rieser et al. [27] showed that the track angle (the angle between the direction of motion and the trajectories of the "tracks" made by body-environment contact) can be modulated by the amplitude of the horizontal wave, $A_{h}$. On granular media, the measure of track angle can give an approximation (a.1)

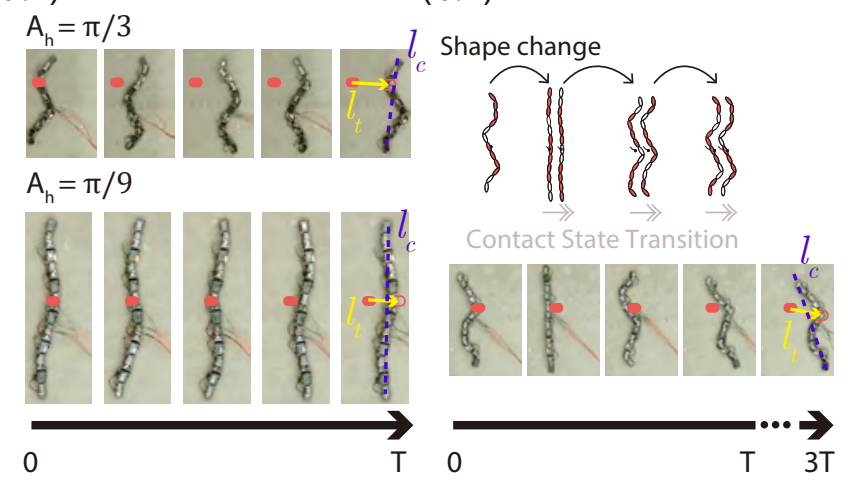

(a.2)

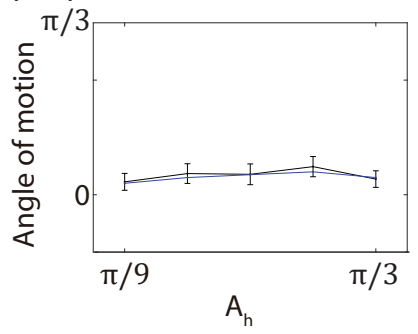

(b.2)

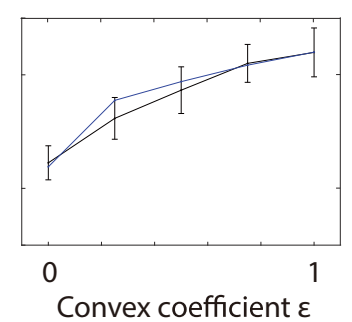

Fig. 5. Experiments on angle of motion modulation. (a.1) Snapshots of robot implementing sidewinding gaits with different amplitudes using sinusoidal templates (Eq. $11 \mid 12$ ). Solid yellow arrow indicates the direction of motion $l_{t}$ and dashed blue line, $l_{c}$ indicate the central body axis. The angle of motion is computed as $\sin ^{-1}\left(\frac{l_{t}^{T} l_{c}}{\left|l_{t}\right| l_{c} \mid}\right)$. (a.2) For the sidewinding gaits using sinusoidal templates, the angle of motion is almost independent of the amplitude for robot moving in isotropic environments. Blue solid line represents simulation and black line with error bars is robophysical experimental data. (b.1) Comparison of snapshots of the robot experiment and the simulation implementing the gait to modulate the angle of motion. (b.2) Modulation of the motion angle by controlling the convex coefficient $\epsilon$.

to the angle of motion (the angle between the direction of motion and the central axis of snake body). However, with our analysis in Sec. II.A.(4), the net displacement of sidewinding gaits on hard ground is predominantly in the lateral direction.

In this way, we tested the sidewinding gaits with a range of amplitudes of the horizontal wave $A_{h}$, from 20 to 60 degrees, on a 12-link limbless robot moving on hard ground. We found through experiments that the angle of motion is almost independent of the amplitude (Fig. 5 a). Example experiment videos for $A_{h}=\pi / 3$ and $\pi / 9$ can be found in the supplementary video. Given the low effectiveness of altering the horizontal amplitude heuristically on the motion angle modulation, we sought to design a general control scheme that would modulate this angle of motion in isotropic environments.

We then applied our method to design sidewinding gaits for a 12-link robot. Following the method introduced in Sec. II, we computed the potential function difference in forward, lateral, and rotation directions (Fig. 6). First, we identified the three transitional points that maximized the displacement in lateral directions, $Q^{y}=\left\{q_{\alpha}^{y}, q_{\beta}^{y}, q_{\gamma}^{y}\right\}$. Note that we limited the joint angle to $\pi / 3$, i.e., $\left\|\left[r_{1}, r_{2}\right]\right\|_{2}<\pi / 3$. We then identified the three transitional points that maximized the displacement 

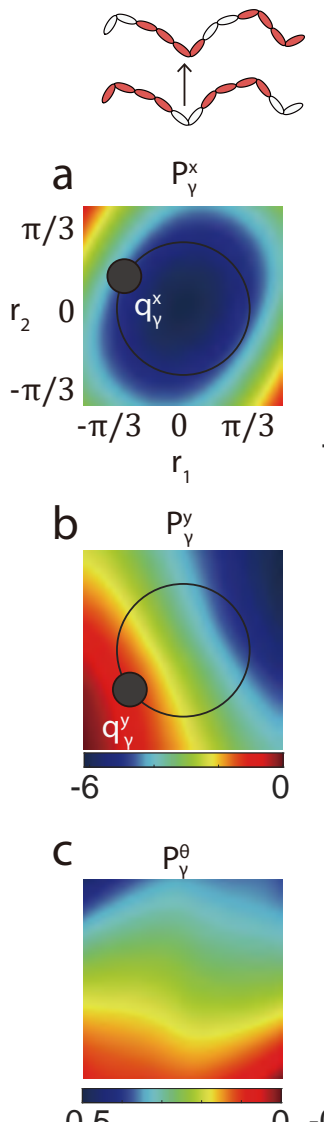

$-0.5$

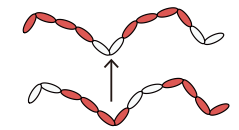

$P_{a}^{x}$
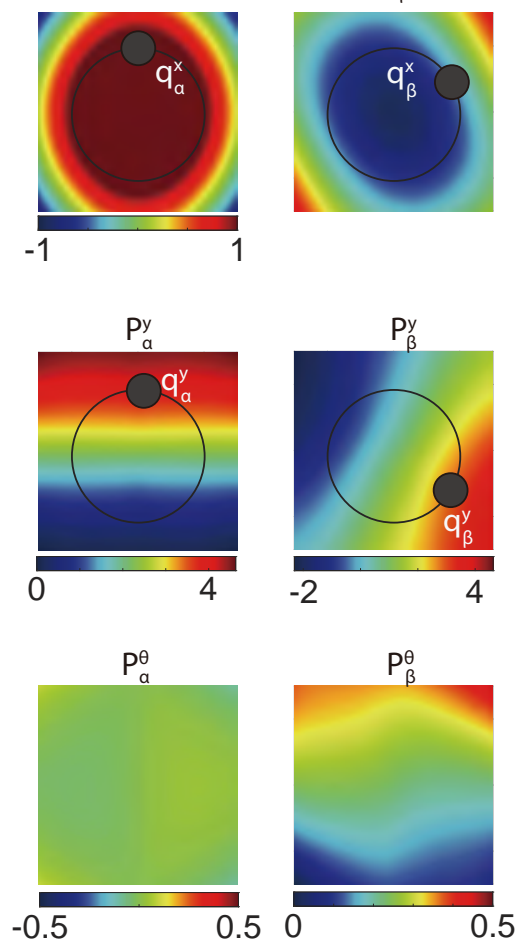

Fig. 6. Modulating the angle of motion using contact pattern optimization. The potential function difference (PFD) in forward (a), lateral (b) and rotational (c) directions. The black circle indicate our joint angle limit: $\left\|\left[r_{1}, r_{2}\right]\right\|_{2} \leq \pi / 3$. The set of extreme points $\left(Q^{x}=\left\{q_{\gamma}^{x}, q_{\alpha}^{x}, q_{\beta}^{x},\right\}\right)$ are chosen to maximize the sum of PFD in forward directions. The set of extreme points $\left(Q^{y}=\left\{q_{\gamma}^{y}, q_{\alpha}^{y}, q_{\beta}^{y},\right\}\right)$ are chosen to maximize the sum of PFD in lateral directions. The axes of all shape spaces are identical. The color bar of PFD in (a) are identical.

in forward direction, $Q^{x}=\left\{q_{\alpha}^{x}, q_{\beta}^{x}, q_{\gamma}^{x}\right\}$. We observed that the transitional points $Q^{y}$ can only lead to pure translation (i.e., zero in forward and rotational directions). Furthermore, the transitional points determined by $Q^{x}$ can lead to effective displacement in both forward and lateral directions, and thus establish a finite angle. In this way, we propose to modulate the angle of motion by a convex combination of $Q^{y}$ and $Q^{x}$ :

$$
Q(\epsilon)=\epsilon Q^{x}+(1-\epsilon) Q^{y},
$$

where $\epsilon \in[0,1]$ is the coefficient of the convex combination, and $Q(\epsilon)$ are the transition points determined by the convex coefficient $\epsilon$.

In this way, using Eq. (13), we formulated the equation to modulate the angle of motion. As shown in Fig $5 \mathrm{~b}$, data from robophysical experiments agreed with our predictions, verifying the validity of our theoretical approach (an example robophysical experiment video can be found in the supplementary video). As such, we have shown that our method is effective in modulating the angle of motion for limbless robots a
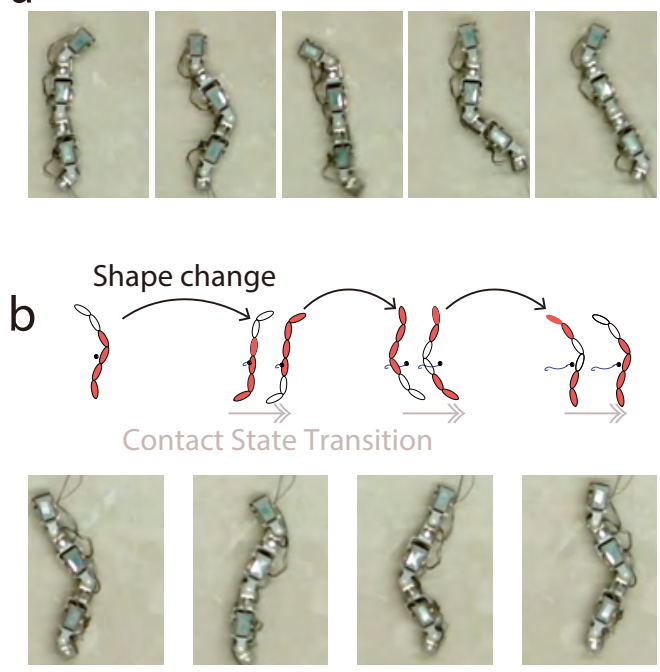

$\mathrm{C}$

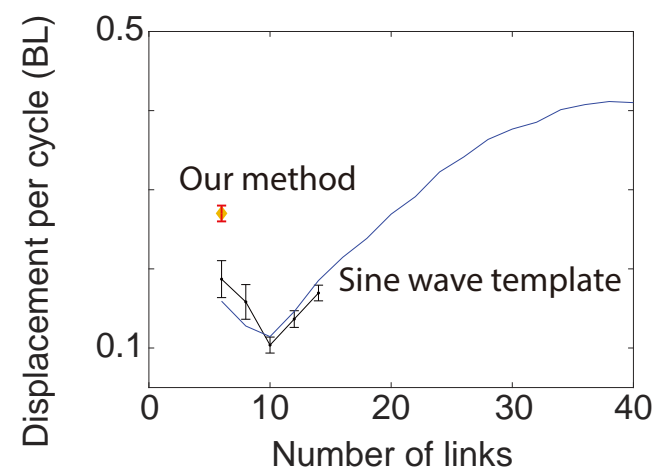

Fig. 7. Sidewinding with fewer number of links. (a) Snapshots of a 6-link robot implementing the sidewinding gait with the sinusoidal templates (Eq. [11, (12)). (b) Snapshots of a 6-link robot implementing the sidewinding gait with our optimization method. (c) The sidewinding speed (in unit BL per cycle) as a function of link numbers (sidewinding gait is prescribed using the sine wave template). Blue solid line represents simulation and black line with error bars is robophysical experimental data. The speed decreases as the link number decreases until $N=10$. For $N<10$, the configuration is unstable and turning emerged. The speed of the gait with our optimization method is highlighted as a diamond marker.

in isotropic environments.

\section{Sidewinding of a 6-link Robot}

While limbless robots have advantages in confined spaces, one of their major restrictions is longitudinal length. In other words, in certain applications such as search and rescue in obstacle-rich environments, it could be desirable to have robots with short body length but high locomotive performance in sidewinding. However, there is often a trade-off between the body length and the locomotive performance for limbless robots: if the size of the motor is fixed, it is only possible to reduce the size of the robots by decreasing the number of motors, i.e., decreasing the degrees of freedom. The disadvantage of fewer motors can be the slower speed of the robot. As shown in [19], even when executing the same gait, robots 
with few motors resulted in significantly less speed than those with adequate motors.

We conducted a series of experiments using the same motion equations but different number of motors. Specifically, we fixed the parameters $A_{h}=\pi / 3, A_{v}=\pi / 9, K=1.5$, and $f=0.1$ and evaluated the relationship between the speed and the number of motors, $N$. The experimental results are shown in Fig.7. As expected, the displacement dropped as the number of motors decreased until $N=10$. Turning behavior emerged at $N<10$, which can be caused by the unstable configurations in the gaits [7]. These unstable turning behaviors led to high variability in their speed, which we attribute to stability. An example of the unstable turning behavior for $N=6$ can be found in the supplementary video.

We then used our method to design the effective sidewinding gaits for the 6-link robot. We first identified three stable contact patterns for this 6-link robot such that the center of mass is enclosed by the supporting polygon. Using the methods introduced in Sec. II, we obtained the potential function difference in lateral direction $\left(P_{\gamma}^{y}, P_{\alpha}^{y}\right.$, and $\left.P_{\beta}^{y}\right)$ and rotational directions $\left(P_{\gamma}^{\theta}, P_{\alpha}^{\theta}\right.$, and $\left.P_{\beta}^{\theta}\right)$. Interestingly, we noticed that the magnitude of $P_{\beta}^{y}$ is significantly lower than those in $P_{\gamma}^{y}$ and $P_{\alpha}^{y}$. Therefore, the lateral speed is almost independent of the choice $q_{\beta}$; and given $q_{\gamma}$ and $q_{\alpha}$, we are free to choose $q_{\beta}$ such that the net rotation is zero.

Given the transitional points, we interpreted the boundary of these contact states as the half line connection origin and chosen transitional points. We can then compute the corresponding vector field and height function.

We implemented our designed gaits in robot experiments. The experimental data shows quantitative agreement with the theoretical predictions. An example video of the 6-link robot experiment can be found in the supplementary video. Interestingly, we noticed that with proper design of the contact pattern, the speed of 6-link robot can even out-perform those with 12 links (Fig. 7 $\mathrm{c}$ ).

\section{Conclusion}

In this paper, we designed a framework to systematically optimize, analyze, and visualize the contact patterns that lead to motion of a limbless robot sidewinding in the desired direction. Specifically, we considered a single contact pattern as a local connection that maps the velocities in the shape space to the position space. We then formulated the optimal contact pattern problem as finding the optimal boundary between each contact state in the shape space. Using the Hodge-Helmholtz theorem, we estimated the line integral in a vector field from its potential functions. By taking the difference in potential functions, we were able to search for the global optimal transitional points in the shape space. Note that in the examples shown in the paper, the curl-free component has a much greater magnitude than the divergence-free component. In cases where the divergencefree and curl-free components have comparable magnitude, we can first determine the transitional points from the curl-free components and design the trajectory connecting transitional points using the divergence-free components.
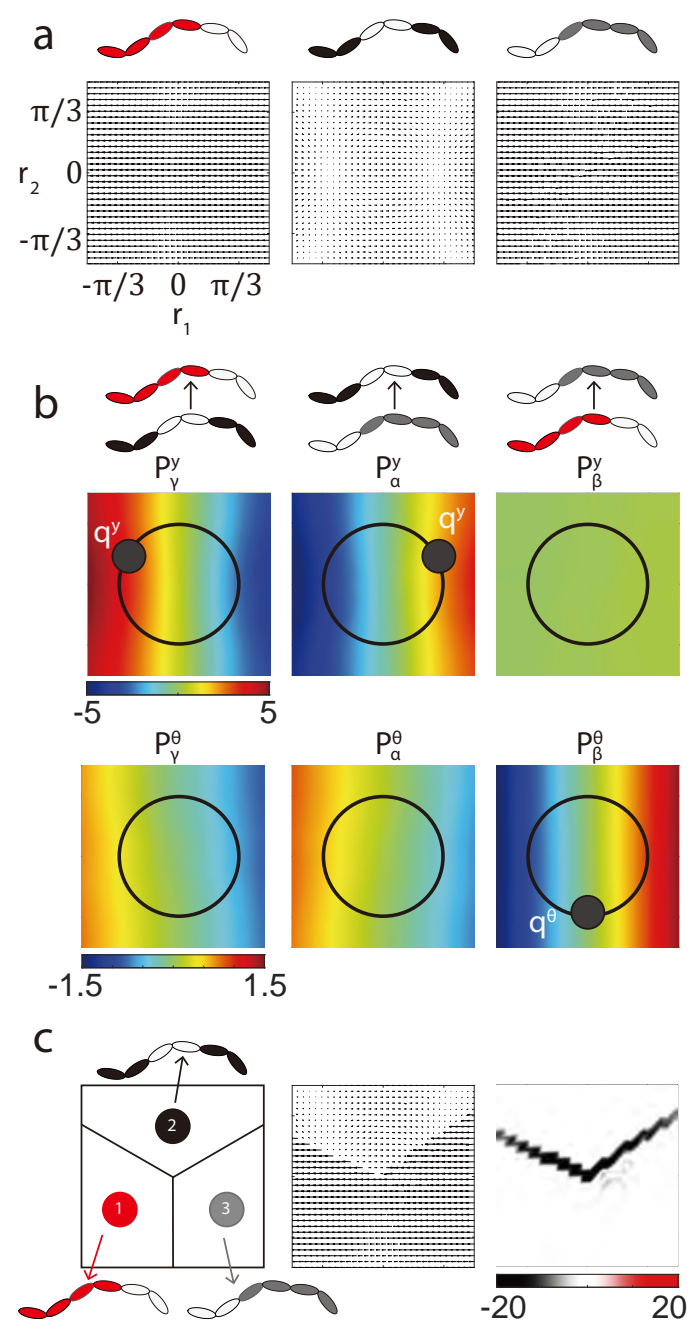

Fig. 8. Designing sidewinding gaits for a 6-link robot. (a) Three stable contact patterns and their corresponding vector fields. (b) The PFD of lateral and rotational directions. The color bars of PFD are identical in three illustrations. The black circle indicates the robot's joint angle limit: $\left\|\left[r_{1}, r_{2}\right]\right\|_{2} \leq \pi / 3$. (c) The boundary of each contact state, the vector field, and the height function with the optimal contact pattern, determined from the obtained transitional points. The unit of color bar in height function is $B L / \pi^{2}$.

We applied our framework to study sidewinding limbless robots. We first used our methods to modulate the angle of motion for sidewinding robots moving in isotropic environments. Robophysical experiments verified that we could modulate the angle of motion by controlling the weights in convex combination $\epsilon$. We then applied our method to designing sidewinding gait for a 6-link robot. We showed that with proper contact design, the 6-link robot can achieve speeds as good as those with adequate number of links (e.g., 12-link snake robot). In this way, we expand the family of sidewinding gaits to robots with fewer motors.

Our future work includes applying our methods to designing optimal contact patterns for legged robots. In addition, we also seek to extend our methods to designing contact patterns for systems with more than 3 contact states. 


\section{REFERENCES}

[1] Jeffrey Aguilar, Tingnan Zhang, Feifei Qian, Mark Kingsbury, Benjamin McInroe, Nicole Mazouchova, Chen Li, Ryan Maladen, Chaohui Gong, Matt Travers, et al. A review on locomotion robophysics: the study of movement at the intersection of robotics, soft matter and dynamical systems Reports on Progress in Physics, 79 (11):110001, 2016.

[2] Silas Alben. Efficient sliding locomotion with isotropic friction Physical Review E, 99(6):062402, 2019.

[3] Henry C Astley, Chaohui Gong, Jin Dai, Matthew Travers, Miguel M Serrano, Patricio A Vela, Howie Choset, Joseph R Mendelson, David L Hu, and Daniel I Goldman. Modulation of orthogonal body waves enables high maneuverability in sidewinding locomotion Proceedings of the National Academy of Sciences, 112(19): 6200-6205, 2015.

[4] Henry C Astley, Jennifer M Rieser, Abdul Kaba, Veronica M Paez, Ian Tomkinson, Joseph R Mendelson, and Daniel I Goldman. Side-impact collision: mechanics of obstacle negotiation in sidewinding snakes Bioinspiration $\mathcal{F}$ Biomimetics, 15(6):065005, 2020.

[5] Robert W Batterman. Falling cats, parallel parking, and polarized light. Studies in History and Philosophy of Science Part B: Studies in History and Philosophy of Modern Physics, 34(4):527-557, 2003.

[6] Baxi Chong, Yasemin Ozkan Aydin, Guillaume Sartoretti, Jennifer M Rieser, Chaohui Gong, Haosen Xing, Howie Choset, and Daniel I Goldman. A Hierarchical Geometric Framework to Design Locomotive Gaits for Highly Articulated Robots In Robotics: science and systems, 2019.

[7] Baxi Chong, Tianyu Wang, Jennifer M Rieser, Abdul Kaba, Howie Choset, and Daniel I Goldman. Frequency Modulation of Body Waves to Improve Performance of Limbless Robots. In Robotics: science and systems, 2020. doi: 10.15607/RSS.2020.XVI.013.

[8] Baxi Chong, Yasemin Ozkan Aydin, Chaohui Gong, Guillaume Sartoretti, Yunjin Wu, Jennifer M Rieser, Haosen Xing, Perrin E Schiebel, Jeffery W Rankin, Krijn B Michel, et al. Coordination of lateral body bending and leg movements for sprawled posture quadrupedal locomotion The International Journal of Robotics Research, page 0278364921991158, 2021.

[9] Robert J Full and Daniel E Koditschek. Templates and anchors: neuromechanical hypotheses of legged locomotion on land. Journal of experimental biology, 202(23): 3325-3332, 1999.

[10] Chaohui Gong, Matthew Travers, Henry C Astley, Daniel I Goldman, and Howie Choset. Limbless locomotors that turn in place In 2015 IEEE International Conference on Robotics and Automation (ICRA), pages 3747-3754. IEEE, 2015.

[11] Chaohui Gong, Daniel I Goldman, and Howie Choset. Simplifying Gait Design via Shape Basis Optimization.
In Robotics: Science and Systems, 2016.

[12] Chaohui Gong, Julian Whitman, Jaskaran Grover, Baxi Chong, Richard Ren, and Howie Choset. Geometric motion planning for systems with toroidal and cylindrical shape spaces. In Dynamic Systems and Control Conference, 2018.

[13] Ross L Hatton and Howie Choset. Nonconservativity and noncommutativity in locomotion. The European Physical Journal Special Topics, 224(17-18):3141-3174, 2015.

[14] Bruce C Jayne. Kinematics of terrestrial snake locomotion. Copeia, pages 915-927, 1986.

[15] Scott D Kelly and Richard M Murray. Geometric phases and robotic locomotion Journal of Field Robotics, 12 (6):417-431, 1995.

[16] Chen Li, Tingnan Zhang, and Daniel I Goldman. A terradynamics of legged locomotion on granular media Science, 339(6126):1408-1412, 2013.

[17] Harvey B Lillywhite. How snakes work: structure, function and behavior of the world's snakes. Oxford University Press, 2014.

[18] Bo Lin, Baxi Chong, Yasemin Ozkan-Aydin, Enes Aydin, Howie Choset, Daniel I Goldman, and Greg Blekherman. Optimizing coordinate choice for locomotion systems with toroidal shape spaces In 2020 IEEE/RSJ International Conference on Intelligent Robots and Systems (IROS), pages 7501-7506, 2020. doi: 10.1109/ IROS45743.2020.9341476.

[19] Ryan D Maladen, Yang Ding, Paul B Umbanhowar, and Daniel I Goldman. Undulatory swimming in sand: experimental and simulation studies of a robotic sandfish The International Journal of Robotics Research, 30(7): 793-805, 2011.

[20] Jerrold E Marsden. Geometric foundations of motion and control. In Motion, Control, and Geometry: Proceedings of a Symposium, Board on Mathematical Science, National Research Council Education, National Academies Press, Washington, DC, 1997.

[21] Jerrold E Marsden and Tudor S Ratiu. Introduction to mechanics and symmetry: a basic exposition of classical mechanical systems, volume 17. Springer Science \& Business Media, 2013.

[22] Hamidreza Marvi, Chaohui Gong, Nick Gravish, Henry Astley, Matthew Travers, Ross L Hatton, Joseph R Mendelson, Howie Choset, David L Hu, and Daniel I Goldman. Sidewinding with minimal slip: Snake and robot ascent of sandy slopes. Science, 346(6206):224229, 2014.

[23] Benjamin McInroe, Henry C Astley, Chaohui Gong, Sandy M Kawano, Perrin E Schiebel, Jennifer M Rieser, Howie Choset, Richard W Blob, and Daniel I Goldman. Tail use improves performance on soft substrates in models of early vertebrate land locomotors Science, 353 (6295):154-158, 2016.

[24] Richard M Murray. A mathematical introduction to robotic manipulation. CRC press, 2017.

[25] Jim Ostrowski and Joel Burdick. The geometric mechan- 
ics of undulatory robotic locomotion The international journal of robotics research, 17(7):683-701, 1998.

[26] Suresh Ramasamy and Ross L Hatton. Soap-bubble optimization of gaits. In 2016 IEEE 55th Conference on Decision and Control (CDC), pages 1056-1062. IEEE, 2016.

[27] Jennifer M Rieser, Chaohui Gong, Henry C Astley, Perrin E Schiebel, Ross L Hatton, Howie Choset, and Daniel I Goldman. Geometric phase and dimensionality reduction in locomoting living systems arXiv preprint arXiv:1906.11374, 2019.

[28] Jennifer M Rieser, Jessica L Tingle, Daniel I Goldman, Joseph R Mendelson, et al. Functional consequences of convergently evolved microscopic skin features on snake locomotion Proceedings of the National Academy of Sciences, 118(6), 2021.

[29] Perrin E Schiebel, Jennifer M Rieser, Alex M Hubbard, Lillian Chen, D Zeb Rocklin, and Daniel I Goldman. Mechanical diffraction reveals the role of passive dynamics in a slithering snake. Proceedings of the National Academy of Sciences, 116(11):4798-4803, 2019.

[30] Perrin E Schiebel, Henry C Astley, Jennifer M Rieser, Shashank Agarwal, Christian Hubicki, Alex M Hubbard, Kelimar Diaz, Joseph R Mendelson III, Ken Kamrin, and Daniel I Goldman. Mitigating memory effects during undulatory locomotion on hysteretic materials. Elife, 9: e51412, 2020.

[31] Alfred Shapere and Frank Wilczek. Geometry of selfpropulsion at low Reynolds number. Journal of Fluid Mechanics, 198:557-585, 1989.

[32] Sarah S Sharpe, Stephan A Koehler, Robyn M Kuckuk, Miguel Serrano, Patricio A Vela, Joseph Mendelson, and Daniel I Goldman. Locomotor benefits of being a slender and slick sand swimmer Journal of Experimental Biology, 218(3):440-450, 2015.

[33] Frank Wilczek and Alfred Shapere. Geometric phases in physics, volume 5. World Scientific, 1989.

[34] Tingnan Zhang and Daniel I Goldman. The effectiveness of resistive force theory in granular locomotion a. Physics of Fluids, 26(10):101308, 2014.

[35] Baxi Zhong, Yasemin Ozkan Aydin, Chaohui Gong, Guillaume Sartoretti, Yunjin Wu, Jennifer Rieser, Haosen Xing, Jeffery Rankin, Krijn Michel, Alfredo Nicieza, et al. Coordination of back bending and leg movements for quadrupedal locomotion. In Robotics: Science and Systems, 2018. 